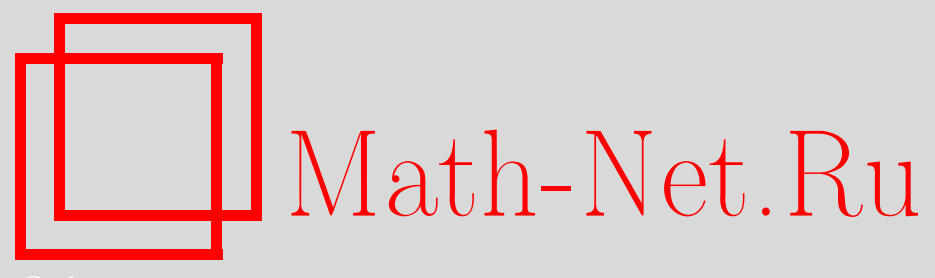

И. А. Круглов, Связь цепей Маркова на конечных простых полугруппах с фундаментальными группами, Дискрет. матем., 2006, том 18, выпуск 2, 48-54

DOI: https://doi.org/10.4213/dm45

Использование Общероссийского математического портала Math-Net.Ru подразумевает, что вы прочитали и согласны с пользовательским соглашением http: //www.mathnet.ru/rus/agreement

Параметры загрузки:

IP : 54.224 .135 .184

26 апреля 2023 г., 14:30:25

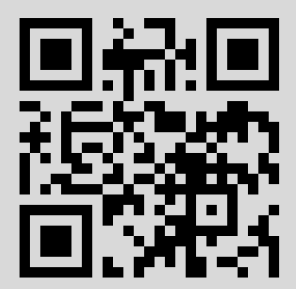




\title{
Связь цепей Маркова на конечных простых полугруппах с фундаментальными группами
}

\author{
๑) 2006 г. И. А. Круглов
}

\begin{abstract}
Исследованы условия сходимости и предельные распределения для последовательностей случайных произведений в конечной простой полугруппе $(S$, о) вида $\eta^{(k)}=s_{\alpha_{1}} \circ s_{\alpha_{2}} \circ \ldots \circ s_{\alpha_{k+1}}$, где $E_{\alpha_{1}}, E_{\alpha_{2}}, \ldots, E_{\alpha_{k+1}}$ - случайная реализация цепочки состояний конечной простой однородной неразложимой цепи Маркова с множеством состояний $\left\{E_{1}, E_{2}, \ldots, E_{n}\right\}, s_{i}, i=1, \ldots, n,-$ фиксированные не обязательно различные элементы $S$. Установлено, что условия сходимости определяются свойствами некоторого гомоморфизма фундаментальной группы графа переходов цепи Маркова в структурную группу полугруппы $S$.

Работа выполнена при поддержке программой Президента Российской Федерации поддержки ведущих научных школ, грант НШ-8564.2006.10.
\end{abstract}

В [1] найдены условия сходимости и предельные распределения для последовательностей произведений случайных величин со значениями в конечных группах, распределения которых определяются конечной простой неразложимой цепью Маркова. В [2] исследованы произведения случайных величин со значениями в конечных простых полугруппах преобразований некоторого конечного множества, распределения которых определяются конечной положительно-регулярной цепью Маркова. Частным случаем таких последовательностей являются последовательности произведений случайных величин, значения которых определяются цепью Маркова. В настоящей работе показано, что в этом частном случае условия сходимости определяются свойствами некоторого гомоморфизма фундаментальной группы графа переходов состояний цепи Маркова в структурную группу простой полугруппы. Это позволяет упростить формулировку условий сходимости, а в некоторых случаях и описание соответствующих предельных распределений. Идея установления связи между предельным поведением изучаемых последовательностей распределений и фундаментальной группой графа цепи Маркова в случае распределений на группах принадлежит Ю. Н. Горчинскому.

Введем необходимые обозначения. Пусть $\mathfrak{I}-$ конечная простая однородная неразложимая цепь Маркова с множеством состояний $\left\{E_{1}, E_{2}, \ldots, E_{n}\right\}$, матрицей переходных вероятностей $P=[p(i, j)]$ и начальным распределением $\left(p_{0}(1), \ldots, p_{0}(n)\right)$. Пусть $(S$, о $)$ - конечная простая полугруппа, $s_{i}, i=1, \ldots, n,-$ элементы полугруппы $S$. По случайной реализации

$$
E_{\alpha_{1}}, E_{\alpha_{2}}, \ldots, E_{\alpha_{k+1}}
$$

цепочки состояний цепи Маркова $\mathfrak{I}$ в первых $k+1$ испытаниях построим произведение

$$
\eta^{(k)}=s_{\alpha_{1}} \circ s_{\alpha_{2}} \circ \ldots \circ s_{\alpha_{k+1}} .
$$


Для натурального $k$ и таких $i, j \in\{1, \ldots, n\}$, что вероятность $\mathbf{P}\left(E_{\alpha_{1}}=E_{i}, E_{\alpha_{k+1}}=E_{j}\right)$ положительна, обозначим через $\eta_{i, j}^{(k)}$ случайную величину, распределение которой совпадает с условным распределением случайной величины $\eta^{(k)}$ при условиях $E_{\alpha_{1}}=E_{i}$, $E_{\alpha_{k+1}}=E_{j}$. В данной работе исследуется предельное поведение последовательностей распределений случайных величин $\eta_{i, j}^{(k)}$.

По теореме Риса-Сушкевича ([3], с. 78) полугруппа $S$ изоморфна рисовской матричной полугруппе $M(G ; A, B ; W)$ над группой $G$ с сандвич-матрицей $W$. Здесь $(G, \cdot)$ - конечная группа (структурная группа $S$ ), $A, B$ - непустые конечные множества, $W=[w(b, a)]_{b \in B, a \in A}$ - матрица с элементами из $G$ размера $|B| \times|A|$. Множество $M(G ; A, B ; W)$ есть множество всех матриц размера $|A| \times|B|$, у которых ровно один элемент есть элемент групшы $G$ и все остальные элементы равны нулю; матрицу размера $|A| \times|B|$ с элементом $g \in G$ в строке с номером $a \in A$ и в столбце номером $b \in B$ будем обозначать $[g ; a, b]$. Бинарная операция в $M(G ; A, B ; W)$ определяется следующим соотношением: если

$$
s^{(i)}=\left[g^{(i)} ; a^{(i)}, b^{(i)}\right] \in M(G ; A, B ; W), \quad i=1,2,
$$

To

$$
s^{(1)} \circ s^{(2)}=\left[g^{(1)} \cdot w\left(b^{(1)}, a^{(2)}\right) \cdot g^{(2)} ; a^{(1)}, b^{(2)}\right]
$$

Всюду далее мы предполагаем, что $S=M(G ; A, B ; W)$, то есть, в отличие от [2], здесь $S$ отождествляется с некоторым ее фиксированным представлением в виде рисовской матричной полугруппы, и формулируем результаты в терминах этого представления.

Мы будем использовать понятия теории графов, следуя изложению п. 3.2 гл. 2 книги [4]. Введем соответствующий цепи $\mathfrak{I}$ граф $\Gamma$ с множеством вершин $\left\{E_{1}, E_{2}, \ldots, E_{n}\right\}$ и множеством ориентированных ребер $\left\{e_{i, j}^{+1}, e_{i, j}^{-1} \mid i, j \in\{1, \ldots, n\}, p(i, j)>0\right\}$. Если $\varepsilon=1$, то $E_{i}$ - начало, а $E_{j}-$ конец $e_{i, j}^{\varepsilon}$, если $\varepsilon=-1$, то $E_{j}-$ начало, а $E_{i}-$ конец $e_{i, j}^{\varepsilon}$. Путем $z$ в графе $\Gamma$ называется последовательность ребер

$$
z=\left(e_{i_{1}, j_{1}}^{\varepsilon_{1}}, e_{i_{2}, j_{2}}^{\varepsilon_{2}}, \ldots, e_{i_{k}, j_{k}}^{\varepsilon_{k}}\right),
$$

у которой для каждого $s=1,2, \ldots, k-1$ начало ребра $e_{i_{s+1}, j_{s+1}}^{\varepsilon_{s+1}}$ совпадает с концом ребра $e_{i_{s}, j_{s}}^{\varepsilon_{s}}$. При этом начало ребра $e_{i_{1}, j_{1}}^{\varepsilon_{1}}$ есть начало пути $z$, конец ребра $e_{i_{k}, j_{k}}^{\varepsilon_{k}}$ - конец пути $z$. Так как цепь Маркова Э неразложима, граф $Г$ сильно связен. Для $i, j \in\{1, \ldots, n\}$ и целого числа $R$ через $L_{R}(i, j)$ обозначим множество всех путей вида (3) в графе $\Gamma$ с началом $E_{i}$ и концом $E_{j}$, у которых $\varepsilon_{1}+\varepsilon_{2}+\ldots+\varepsilon_{k}=R$. Пусть

$$
Z^{(1)}=\bigcup_{R=-\infty}^{\infty} L_{R}(1,1)
$$

- множество петель в $\Gamma$ с концом $E_{1}, \Phi(\Gamma)$ - множество классов эквивалентных петель с концом $E_{1}$, множество $\Phi(\Gamma)$ относительно операции произведения классов петель является группой и называется фундаментальной группой графа $Г$ (см. [4]). Для петли $z \in Z^{(1)}$ будем также через $z$ обозначать ее класс как элемент $\Phi(\Gamma)$.

Пусть

$$
\begin{aligned}
s_{i} & =\left[g_{i} ; a_{i}, b_{i}\right], \quad g_{i} \in G, \quad a_{i} \in A, \quad b_{i} \in B, \quad i=1, \ldots, n, \\
g_{i, j} & =g_{i} \cdot w\left(b_{i}, a_{j}\right), \quad p(i, j)>0, \quad i, j \in 1, \ldots, n
\end{aligned}
$$


Пути $z$ вида (3) поставим в соответствие элемент $g(z)=g_{i_{1}, j_{1}}^{\varepsilon_{1}} \cdot g_{i_{2}, j_{2}}^{\varepsilon_{2}} \cdot \ldots \cdot g_{i_{k}, j_{k}}^{\varepsilon_{k}}$ группы $G$. Если путь $z$ есть произведение $(u, v)$ путей $u$ и $v$, то $g(z)=g(u) \cdot g(v)$. Если пути $u$ и $v$ эквивалентны, то $g(u)=g(v)$. Отсюда следует, что отображение $\varphi: \Phi(\Gamma) \rightarrow G$, где $\varphi(z)=g(z)$ для всех $z \in \Phi(\Gamma)$, определено корректно и является гомоморфизмом групп. Обозначим через $G_{1}$ образ группы $\Phi(\Gamma)$ при гомоморфизме $\varphi$.

Пусть $d$ - число циклических подклассов (неразложимой) цепи Маркова Э. Для петли $z \in Z^{(1)}$ вида (3) положим

$$
l(z)=\frac{1}{d}\left(\varepsilon_{1}+\varepsilon_{2}+\ldots+\varepsilon_{k}\right) .
$$

Нетрудно видеть, что $l(z) \in \mathbf{Z}$ для всех $z \in Z^{(1)}$. Если петли $z$ и $u$ эквивалентны, то $l(z)=l(u)$, если петля $z$ есть произведение петель $u$ и $v$, то $l(z)=l(u)+l(v)$. Таким образом, отображение $l$ определяет гомоморфизм группы $\Phi(\Gamma)$ в группу $(\mathrm{Z},+)$, который мы также будем обозначать через $l$. Кроме того, поскольку $d$ есть наибольший общий делитель множества чисел $\left\{d l(z) \mid z \in Z^{(1)}\right\}$, отображение $l: \Phi(\Gamma) \rightarrow \mathbf{Z}$ есть эпиморфизм. Зафиксируем произвольно $z^{\prime} \in \Phi(\Gamma)$, для которого $l\left(z^{\prime}\right)=1$, пусть $x_{1}=\varphi\left(z^{\prime}\right)$. Пусть $\Phi_{0}=\operatorname{Ker} l, H=\varphi\left(\Phi_{0}\right)$, тогда $H$ - нормальный делитель $G_{1}$, а $x_{1} \cdot H$ - образующий элемент фактор-группы $G_{1} / H$. Пусть $r_{1}$ - индекс $H$ в $G_{1}$.

Обозначим через $\varepsilon_{\Phi_{0}}$ отображение вложения $\Phi_{0}$ в $\Phi(Г)$, через $\varepsilon_{H}$ - отображение вложения $H$ в $G_{1}$, через $\rho_{H}$ - естественный эпиморфизм группы $G_{1}$ на фактор-группу $G_{1} / H$, а также пусть $\lambda_{r_{1}}: Z \rightarrow G_{1} / H-$ эпиморфизм, при котором

$$
\lambda_{r_{1}}(m)=x_{1}^{m} \cdot H, \quad m \in \mathbf{Z} .
$$

Тогда имеет место следующая коммутативная диаграмма гомоморфизмов групп с точными последовательностями в строках и столбцах:

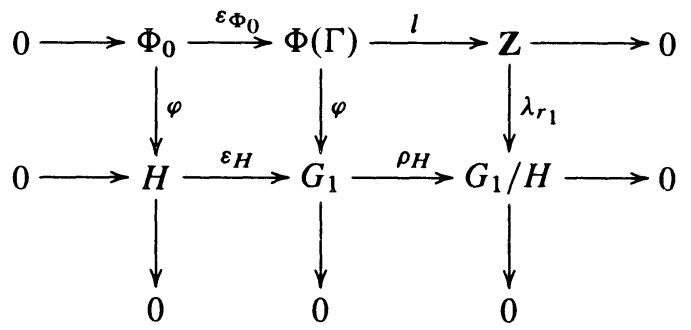

Любой паре $i, j \in\{1, \ldots, n\}$ однозначно соответствует число $v(i, j) \in\{0,1, \ldots, d-1\}$, для которого $L_{R}(i, j)$ непусто при $R=v(i, j)+k d$ и любом целом $k$. Пусть

$$
\gamma(i, j)=\frac{1}{d}(v(1, i)+v(i, j)-v(1, j)),
$$

очевидно, что $\gamma(i, j) \in\{0 ;+1\}, i, j \in\{1, \ldots, n\}$. Зафиксируем произвольно $z_{i, j} \in L_{v(i, j)}(i, j)$, и пусть $\sigma_{i, j}=g\left(z_{i, j}\right), i, j \in\{1, \ldots, n\}$.

Теорема 1. Пусть фиксированы некоторые $i, j \in\{1, \ldots, n\}$, причем $p_{0}(i)>0, a\left\{k_{N}\right\}$, $N=1,2, \ldots,-$ последовательность натуральных чисел, $k_{N} \rightarrow \infty$ при $N \rightarrow \infty$. Тогда при $N \rightarrow \infty$ последовательность распределений случайных величин

$$
\eta_{i, j}^{\left(s_{N}\right)}, \quad s_{N}=v(i, j)+k_{N} d
$$


имеет предел тогда и только тогда, когда существует $l \in\left\{0,1, \ldots, r_{1}-1\right\}$, для которого $k_{N} \equiv l\left(\bmod r_{1}\right)$ при всех достаточно больших $N$. При выполнении этого условия последовательность векторов распределений случайных величин (4) сходится к вектору равновероятного распределения на множестве $\left\{\left[g ; a_{i}, b_{j}\right], g \in \sigma_{1, i}^{-1} \cdot x_{1}^{l+\gamma(i, j)} \cdot H \cdot \sigma_{1, j} \cdot g_{j}\right\}$.

Доказательство. Рассмотрим схему произведений случайных величин со значениями в группе $G$ (см. [1], §3), в которой случайной реализации (1) цепи Маркова $\mathfrak{I}$ соответствует случайная величина $\tilde{\eta}^{(k)}=g_{\alpha_{1}, \alpha_{2}} \cdot g_{\alpha_{2}, \alpha_{3}} \cdot \ldots \cdot g_{\alpha_{k}, \alpha_{k+1}}$. Пусть $\tilde{\eta}_{i, j}^{(k)}$ - случайная величина со значениями в $G$, распределение которой есть условное распределение $\tilde{\eta}^{(k)}$ при условиях $E_{\alpha_{1}}=E_{i}, E_{\alpha_{k+1}}=E_{j}$. Тогда из соотношения (2) следуют равенства

$$
\eta^{(k)}=\left[\tilde{\eta}^{(k)} \cdot g_{\alpha_{k+1}} ; a_{\alpha_{1}}, b_{\alpha_{k+1}}\right], \quad \eta_{i, j}^{(k)}=\left[\tilde{\eta}_{i, j}^{(k)} \cdot g_{j} ; a_{i}, b_{j}\right]
$$

Для исследования предельного поведения последовательностей $\tilde{\eta}_{i, j}^{(k)}$ можно использовать результаты работы [1]. Заметим, что подгруппа $G_{1}$, элементы $x_{1}, \sigma_{i, j}$ и числа $\nu(i, j)$, $i, j \in\{1, \ldots, n\}$, совпадают с определенными в $\$ 3$ работы [1] подгруппой, элементами $G$ и числами, имеющими те же обозначения. Кроме того, в [1] вводится минимальная подгруппа $H_{1}$ группы $G$ среди ее подгрупп $L$, удовлетворяющих соотношению

$$
g(z) \in x_{1}^{l(z)} \cdot L \quad \forall z \in Z^{(1)} .
$$

Докажем, что $H_{1}=H=\varphi\left(\Phi_{0}\right)$. Пусть $\tau \in H$, тогда $\tau=g(z)$ для некоторого $z \in Z^{(1)}$ такого, что $l(z)=0$. Согласно соотношению (6), выполненному при $L=H_{1}$, имеет место включение $\tau \in H_{1}$. Следовательно, $H \subset H_{1}$. Пусть $z \in Z^{(1)}$, тогда $u=\left(z,\left(z^{\prime}\right)^{-l(z)}\right) \in Z^{(1)}$, $l(u)=0, \varphi(u)=g(z) \cdot x_{1}^{-l(z)} \in H$ и $g(z) \in x_{1}^{l(z)} \cdot H$. Таким образом, (6) выполнено при $L=H$, тогда, в силу минимальности, $H_{1} \subset H$. Итак, $H=H_{1}$. В силу основной теоремы $\S 3$ работы [1] последовательность распределений случайных величин $\tilde{\eta}_{i, j}^{\left(s_{N}\right)}$ имеет предел при $N \rightarrow \infty$, если и только если существует $l \in\left\{0,1, \ldots, r_{1}-1\right\}$, для которого $k_{N} \equiv l$ $\left(\bmod r_{1}\right)$ для всех достаточно больших $N$, и при выполнении этого условия предельное распределение есть равновероятное распределение на множестве $\sigma_{1, i}^{-1} \cdot x_{1}^{l+\gamma(i, j)} \cdot H_{1} \cdot \sigma_{1, j}$. С учетом (5) отсюда следует утверждение доказываемой теоремы.

Множество

$$
H_{a_{i}, b_{j}}=\left\{\left[g ; a_{i}, b_{j}\right] \mid g \in G\right\}
$$

есть $H$-класс полугруппы $S$, являющийся пересечением ее $R$-класса с номером $a_{i}$ и $L$-класса с номером $b_{j}$. Из соотношения (5) следует, что множество

$$
\left\{s \in S \mid \mathbf{P}\left(\eta_{i, j}^{(k)}=s\right)>0\right\} \subset H_{a_{i}, b_{j}},
$$

если $\mathbf{P}\left(E_{\alpha_{1}}=E_{i}, E_{\alpha_{k+1}}=E_{j}\right)>0$. В связи с этим представляет интерес следуюшее утверждение.

Следствие 1. Для произвольных $i, j \in\{1, \ldots, n\}, p(i)>0$, последовательность векторов распределений случайных величин $\eta_{i, j}^{\left(s_{N}\right)}, s_{N}=v(i, j)+N \cdot d, N \geqslant 1$, сходится $\kappa$ вектору равновероятного распределения на множестве $H_{a_{i}, b_{j}}$ тогда и только тогда, когда отображение $\varphi: \Phi_{0} \rightarrow G$ сюръективно. 
Условие сюръективности $\varphi$ на множестве $\Phi_{0}$ можно переформулировать в терминах порождаемости группы $G$ некоторым подмножеством ее элементов. Пусть $t$ - число ненулевых элементов матрищы переходных вероятностей $P$. Согласно [4], группа $\Phi(\Gamma)$ есть свободная группа ранга $(t-n+1)$, в [4] приведен способ выбора $t-n+1$ свободных образуюших $\Phi(\Gamma)$, основанный на выделении в $\Gamma$ максимального подграфа, являющегося деревом с корнем $E_{1}$.

Пусть $\pi_{1}, \ldots, \pi_{c} \in \Phi(\Gamma)$ - произвольная система не обязательно свободных образующих группы $\Phi(\Gamma)$. Тогда нетрудно показать, что множество $\left\{g\left(\pi_{1}\right), \ldots, g\left(\pi_{c}\right)\right\}$ порождает групту $G_{1}$, а множество

$$
A=\left\{x_{1}^{k} \cdot g\left(\pi_{i}\right) \cdot x_{1}^{-k-l\left(\pi_{i}\right)}, i=1,2, \ldots, c, k=0,1, \ldots, \text { ord } x_{1}-1\right\}
$$

порождает группу $H$.

Следствие 2. Для произвольных $i, j \in\{s, \ldots, n\}, p_{0}(i)>0$, последовательность векторов распределений случайных величин $\eta_{i, j}^{\left(s_{N}\right)}, s_{N}=v(i, j)+N \cdot d, N \geqslant 1$, сходится $\kappa$ вектору равновероятного распределения на множестве $H_{a_{i}, b_{j}}$ тогда и только тогда, когда множество $A$ порождает группу $G$.

Рассмотрим теперь частный случай, когда простая полугруппа $S$ является группой, то есть $(S, \circ)=(G, \cdot), A=\{a\}, B=\{b\}, w(b, a)=e_{G}-$ единичный элемент $G, s_{i}=g_{i}$ для всех $i=1, \ldots, n, g_{i, j}=g_{i}, i, j \in\{1, \ldots, n\}, p(i, j)>0$.

В этом случае последовательность $\eta^{(k)}$ есть последовательность произведений случайных величин с значениями в конечной группе $G$, связанных в цепь Маркова или в случайный процесс, полученный объединением состояний цепи Маркова. Будем также предполагать, что цепь Маркова $\mathfrak{I}$ положительно регулярна, то есть $d=1$. В этом случае $v(i, j)=\gamma(i, j)=0$ для всех $i, j \in\{1, \ldots, n\}, l(z)=\varepsilon_{1}+\ldots+\varepsilon_{k}$ для любого $z \in Z^{(1)}$ вида (3).

Введем условие на цепь Маркова $\mathfrak{3}$, в значительной степени упрощающее исследование предельного поведения последовательностей распределений случайных величин $\eta_{i, j}^{(k)}$. Пусть $\left(F_{n}, *\right)$ - свободная группа ранга $n$ с множеством свободных образующих $f_{1}, f_{2}, \ldots, f_{n}$. Аналогично гомоморфизму $\varphi$ можно ввести гомоморфизм групп $\chi: \Phi(\Gamma) \rightarrow F_{n}$, полагая для $z \in Z^{(1)}$ вида (3)

$$
\chi(z)=f_{i_{1}}^{\varepsilon_{1}} * f_{i_{2}}^{\varepsilon_{2}} * \ldots * f_{i_{k}}^{\varepsilon_{k}} .
$$

Введем обозначение

$$
\Omega=\left\{g_{1}, g_{2}, \ldots, g_{n}\right\}
$$

Следствие 3. Пусть $\chi$ - эпиморфизм групп. Тогда

(1) подгруппа $G_{1}$ есть подгруппа группы $G$, порожденная множеством $\Omega$; $Н$ есть минимальный нормальный делитель группы $G_{1}$ среди всех ее нормальных делителей $L$, удовлетворяющих условию $\Omega \subset g_{1} \cdot L$;

(2) для любьх $i, j \in\{1,2, \ldots, n\}, p_{0}(i)>0$, последовательность векторов распределений случайных величин $\eta_{i, j}^{\left(k_{N}\right)}$ имеет предел при $N \rightarrow \infty$ тогда и только тогда, когда существует $l \in\left\{0,1, \ldots, r_{1}-1\right\}$ такое, что $k_{N} \equiv l\left(\bmod r_{1}\right)$ для всех достаточно больших $N$, при этом предельный вектор есть вектор равновероятного распределения на множестве $g_{1}^{l} \cdot H$. 
Доказательство. Докажем первую часть. Обозначим через $G_{2}$ подгруппу в $G$, порожденную $\Omega$, и докажем, что $G_{2}=G_{1}$. Очевидно, что $G_{1} \subset G_{2}$. Для доказательства обратного включения достаточно показать, что $\Omega \subset G_{1}$. Фиксируем $i \in\{1, \ldots, n\}$, так как $\chi-$ эпиморфизм, существует $z \in Z^{(1)}$ вида (3), для которого

$$
\chi(z)=f_{i_{1}}^{\varepsilon_{1}} * \ldots * f_{i_{k}}^{\varepsilon_{k}}=f_{i}
$$

Отображение $\lambda: f_{i} \rightarrow g_{i}, i \in\{1, \ldots, n\}$, индуцирует гомоморфизм $\lambda: F_{n} \rightarrow G$, причем $\varphi=\chi \circ \lambda$. Следовательно,

$$
\varphi(z)=\lambda\left(f_{i_{1}}^{\left(\varepsilon_{1}\right)} * \ldots * f_{i_{k}}^{\left(\varepsilon_{k}\right)}\right)=\lambda\left(f_{i}\right)=g_{i} .
$$

Таким образом, $g_{i} \in G_{1}$ для всех $i \in\{1, \ldots, n\}$, что и требовалось доказать.

Обозначим через $H_{2}$ минимальный нормальный делитель группы $G_{1}$ среди всех еe нормальных делителей $L$, удовлетворяющих соотношению $\Omega \subset g_{1} * L$, докажем, что $H_{2}=H$. Группа $G_{1} / H_{2}$ циклическая, порожденная смежным классом $g_{1} \cdot H_{2}$. Докажем, что $H \subset H_{2}$. Для любой петли $z \in Z^{(1)}$ вида (3)

$$
\varphi(z)=g(z)=g_{i_{1}}^{\varepsilon_{1}} \cdot \ldots \cdot g_{i_{k}}^{\varepsilon_{k}} \in g_{1}^{l(z)} \cdot H_{2} .
$$

Следовательно, $H=\varphi\left(\Phi_{0}\right) \subset H_{2}$. Для доказательства включения $H_{2} \subset H$ достаточно показать, что $\Omega \subset g_{1} * H$. Фиксируем $i \in\{1, \ldots, n\}$ и выберем петлю $z \in Z^{(1)}$ вида (3), удовлетворяющую условию (7). Из (7) следует, что $l(z)=1$. Пусть $u=\left(z,\left(z^{\prime}\right)^{-1}\right)$, тогда $\varphi(u)=\varphi(z) \cdot \varphi\left(z^{\prime}\right)^{-1}=g_{i} \cdot x_{1}^{-1}, l(u)=0, \varphi(u) \in \varphi\left(\Phi_{0}\right)=H$ и $g_{i} \in x_{1} \cdot H$ для всех $i \in\{1, \ldots, n\}$. Но тогда $\Omega \subset g_{1} * H$.

Докажем вторую часть. В силу только что доказанной первой части, для любых $i, j \in\{1, \ldots, n\}, R \in \mathbf{Z}, z \in L_{R}(i, j)$ верно включение $g(z) \in g_{1}^{R} \cdot H$. Следовательно, $x_{1} \in g_{1} \cdot H, \sigma_{i, j} \in H$ для всех $i, j \in\{1, \ldots, n\}$. Поэтому справедливость второй части следствия непосредственно вытекает из утверждения теоремы.

Заметим, что при выполнении условия сюръективности $\chi$ предельное поведение последовательностей $\eta_{i, j}^{(N)}$ совпадает с предельным поведением произведений независимых одинаково распределенных случайных величин, у которых множество значений в $G$, принимаемых с положительной вероятностью, есть множество $\Omega$. В этом смысле условие сюръективности $\chi$ есть условие предельной слабой зависимости случайных величин со значениями в конечной группе, связанных в функцию от состояний цепи Маркова 3.

Пусть $\left(A_{n},+\right)$ - свободная абелева группа ранга $n$ с множеством свободных образующих $\left\{a_{1}, \ldots, a_{n}\right\}$. Аналогично определению гомоморфизма $\varphi$, определим гомоморфизм $\psi: \Phi(\Gamma) \rightarrow A_{n}$, полагая для всех $z \in Z^{(1)}$ вида (3)

$$
\psi(z)=\varepsilon_{1} \cdot a_{1}+\varepsilon_{2} \cdot a_{2}+\ldots+\varepsilon_{n} \cdot a_{n}
$$

В случае, когда $G$ - конечная абелева группа, утверждение следствия 3 останется справедливым, если условие сюръективности отображения $\chi$ заменить на условие сюръективности $\psi$.

Условие сюръективности $\psi$ совпадает с известным условием А. Н. Колмогорова [5] на цепь Маркова $\mathfrak{I}$, обеспечивающим асимптотическую нормальность нормированных сумм вещественных случайных величин, определяемых цепью Маркова Э. Нетрудно видеть, что из сюръективности гомоморфизма $\chi$ следует сюръективность гомоморфизма $\psi$. Таким 
образом, условие сюръективности $\chi$ можно рассматривать в качестве аналога условия А. Н. Колмогорова для произвольных конечных групп.

В заключение приведем условие на матрицу $P$, равносильное сюръективности $\chi$. Пусть $P^{\prime}$ - матрица, транспонированная к $P$.

Предложение 1. Пусть $P$ - неразложимая матрича. Тогда $\chi$ сюръективен тогда и только тогда, когда матрица $P^{\prime} P$ неразложима.

Доказательство. Пусть матрица $P^{\prime} P$ неразложима. Необходимо доказать, что для каждого $i \in\{1, \ldots, n\}$ существует $v \in Z^{(1)}$ такой, что $\chi(v)=f_{i}$. Фиксируем $i \in\{1, \ldots, n\}$ и выберем $j \in\{1, \ldots, n\}$ такое, что $p(i, j)>0$. Так как $P^{\prime} P$ неразложима, существуют пути

$$
\begin{aligned}
& v_{1}=\left(e_{i_{1}, 1}^{-1} ; e_{i_{1}, j_{2}}^{+1} ; e_{i_{3}, j_{2}}^{-1} ; e_{i_{3}, j_{4}}^{+1} ; \ldots ; e_{i_{q}, j_{q-1}}^{-1} ; e_{i_{q}, i}^{+1}\right) \in L_{0}(1, i), \\
& v_{2}=\left(e_{t_{1}, j}^{-1} ; e_{t_{1}, k_{2}}^{+1} ; \ldots ; e_{t_{l}, k_{l-1}}^{-1} ; e_{t_{l}, 1}^{+1}\right) \in L_{0}(j, 1) .
\end{aligned}
$$

Тогда путь $v=\left(v_{1}, e_{i, j}^{+1}, v_{2}\right) \in Z^{(1)}$ удовлетворяет условию $\chi(v)=f_{i}$.

Пусть $\chi$ сюръективно, докажем, что матрица $P^{\prime} \cdot P$ неразложима. Достаточно доказать, что для каждого $i \in\{2,3 \ldots, n\}$ существует путь $v_{1}$ вида (8). Так как $\chi$ - эпиморфизм, существует путь $z \in Z^{(1)}$, для которого $\chi(z)=f_{i}$. Будем предполагать, что $z$ есть путь с минимальным числом ребер, удовлетворяющий данному условию. Пусть $z$ имеет вид (3), тогда $\chi(z)=f_{i}=f_{i_{1}}^{\varepsilon_{1}} * \ldots * f_{i_{k}}^{\varepsilon_{k}}$. Рассмотрим последовательность редуцированных слов $W_{1}, \ldots, W_{k}$, полученную применением $W$-процесса к слову $f_{i_{1}}^{\varepsilon_{1}}, \ldots, f_{i_{k}}^{\varepsilon_{k}}$ в образующих $f_{1}, \ldots, f_{k}$ (см. [6], §7.1). Напомним, что $W_{1}=f_{i_{1}}^{\varepsilon_{1}}$, и если $W_{1}, \ldots, W_{\nu}$ определены для $\nu \in\{1, \ldots, k-1\}$, то в случае, когда $W_{\nu}=\left(V, f_{i_{v+1}}^{-\varepsilon_{v+1}}\right)$ для некоторого слова $V, W_{\nu+1}=V$, в противном случае $W_{v+1}=\left(W_{\nu}, f_{i_{v+1}}^{\varepsilon_{v+1}}\right)$. Из леммы 7.1.1 в [6] следует, что $W_{k}=f_{i}$. Пусть $m \in\{1, \ldots, k\}$ - минимальное число, удовлетворяющее свойству $W_{m}=f_{i}$, так как $i \neq 1$, то $m>1$. Используя свойство минимальности числа ребер в пути $z$, нетрудно показать, что $m$ нечетно и $\varepsilon_{1}=-1, \varepsilon_{2}=+1, \ldots, \varepsilon_{m-2}=-1, \varepsilon_{m-1}=+1, \varepsilon_{m}=+1, i_{m}=i$. Но тогда $j_{1}=1, j_{m-1}=i$, и путь $e_{i_{1}, j_{1}}^{\varepsilon_{1}}, \ldots, e_{i_{m-1}, j_{m-1}}^{\varepsilon_{m-1}}$ имеет вид (8) при $q=m-1$.

Предложение доказано.

Следствие 4. Если матриџы $P$ и $P^{\prime} P$ неразложимы, то иепь Маркова $\mathfrak{\Im}$ удовлетворяет условию А. Н. Колмогорова.

\section{Список литературы}

1. Горчинский Ю. Н., Круглов И. А., Капитонов В. М., Вопросы теории распределений на конечных группах. Труды по дискретной математике (1997) 1, 85-112.

2. Алиев $\Phi$. К., О предельных распределениях произведений случайных величин на конечной простой полугруппе с распределениями, заданными на переходах положительно-регулярной цепи Маркова. Обозрение прикладной и промышленной математики (1997) 4, №3, 316-318.

3. Лаллеман Ж., Полугруппы и комбинаторные приложения. Мир, Москва, 1985.

4. Бахтурин Ю. А., Основные структуры современной алгебры. Наука, Москва, 1990.

5. Сарымсаков Т. А., Основы теории прочессов Маркова. ГИТТЛ, Москва, 1954.

6. Холл М., Теория групn. ИЛ, Москва, 1962. 